\title{
WILEY-VCH
}

DOI: 10.1002/ ((please add manuscript number))

Article type: Communication

\section{Core-Shell Upconversion Nanoparticle@Metal-Organic Framework Nanoprobes for Luminescent/Magnetic Dual-mode Targeted Imaging}

Yantao Li, ${ }^{\dagger}$ Jinglong Tang, ${ }^{\dagger}$ Liangcan He, Yong Liu, Yaling Liu,* Chunying Chen, * and Zhiyong Tang*

Y. Li, J. Tang, L. He, Y. Liu, Prof. Y. Liu, Prof. C. Chen, Prof. Z. Tang

Key Laboratory of Nanosystem and Hierarchical Fabrication, Chinese Academy of Sciences

National Center for Nanoscience and Technology

No.11, Beiyitiao, Zhongguancun, Beijing, 100190, P. R. China

E-mail: liuyl@nanoctr.cn; chenchy@nanoctr.cn; zytang@nanoctr.cn

$\dagger$ These authors contributed equally.

Keywords: core-shell UCNP@Fe-MIL-101_NH $\mathrm{NH}_{2}$ nanostructures, surface modification,

UCL/MRI dual-mode targeted imaging, biocompatibility

Abstract: Multifunctional core-shell nanostructures made of inorganic nanoparticles (NPs) integrated with metal-organic frameworks (MOFs) have attracted much attention in recent years. In this work, well-defined core-shell upconversion NP@MOF nanostructures are constructed by coating hexagonal $\mathrm{NaYF}_{4}$ :Yb,Er NP cores with amino-functionalized iron carboxylate MOF shells. Notably, these nanostructures display good water dispersibility, low toxicity, and facile conjugation with PEG and folic acid. For the first time, these novel coreshell NP@MOF nanostructures are successfully tested both in vitro and in vivo for tumortargeted luminescence/MR imaging.

Porous materials have been widely used in biotechnology, including imaging, sensing, genomics, diagnostics, and therapy. ${ }^{[1]}$ Compared with classic porous organic (carbons) or inorganic counterparts (zeolites, silica), metal-organic frameworks (MOFs), composed of metal ions or clusters connected to each other by polydentate bridging ligands, have tunable composition, easy functionalization, accessible metal sites and high capacity for drug or gas 


\section{WILEY-VCH}

loading, and good biocompatibility and biodegradability. ${ }^{[2]}$ When MOF materials have been scaled down to form nanoscale metal-organic frameworks (NMOFs), they will maintain the properties of bulk MOFs and also exhibit the nanoscale characteristics, making them as a promising platform for biomedical applications. ${ }^{[3]}$ More specialized investigations have recently focused on synthesis of NMOFs and their use in bioimaging and drug delivery. ${ }^{[4]}$ Especially, the MIL family ${ }^{[5]}$ built from non-toxic iron carboxylate NMOFs (MIL-88A, MIL100, MIL-101_ $\mathrm{NH}_{2}$ ), is widely reported to exhibit high capacity drug loading, progressive release, and $\mathrm{T}_{2}$-weighted magnetic resonance imaging properties with low toxicity, which makes them good candidates for biomedical applications. However, there are only a few reports about the functionalization of NMOFs or their complexes for targeted imaging or therapy (either in vitro or in vivo), which would have great significance in further developments. ${ }^{[3]}$

Rare-earth doped upconversion nanoparticles (UCNPs) have become promising candidates for constructing multi-modal nanoprobes owing to their excellent near-infrared (NIR) excited upconversion luminescence (UCL) properties. ${ }^{[6]}$ Among the multi-modal bioimaging systems of UCNPs, integration of UCL and MRI in one system may combine deep tissue penetration, high photo-stability, and low optical background of NIR imaging with the good dimensional resolution of MRI. ${ }^{[7]}$ UCNP based UCL/MRI imaging agents are either $\mathrm{T}_{1}$ or $\mathrm{T}_{2}$ contrast agents, UCL/ $\mathrm{T}_{1}$-MRI and UCL/ $\mathrm{T}_{2}$-MRI, respectively. Due to the seven unpaired inner $4 \mathrm{f}$ electrons, $\mathrm{Gd}^{3+}$ based UCNPs have been developed as the most useful $\mathrm{T}_{1}$-weighted MRI agents. Also, prototypes of UCNPs coupled with superparamagnetic iron oxide NPs (SPION) have been widely applied in $\mathrm{UCL} / \mathrm{T}_{2}$-MRI contrast agents because of their large magnetic moment. ${ }^{[8]}$ However, there are still many challenges needed to be addressed for these systems, such as the toxicity of $\mathrm{Gd}^{3+}$ ions, and the destabilization and agglomeration of the core-shell UCNP@Fe $\mathrm{O}_{\mathrm{y}} \mathrm{NPs}$, which may precludes their clinical applications. ${ }^{[9]}$ Consequently, there is 


\section{WILEY-VCH}

an emergent requirement to develop novel UCL/MRI contrast agents with easier preparation and functionalization, less biotoxicity, better biocompatibility, and biodegradability.

It is worth mentioning that recent work has successfully integrated UCNPs and MOFs into the well-defined structure via encapsulation of UCNPs within MOFs. ${ }^{[10]}$ Inspired by this, here we adopt a different strategy, that is, combination of single $\mathrm{NaYF}_{4}$ :Yb,Er UCNP core into FeMIL-101_NH2 $\mathrm{NMOF}^{[11]}$ shell, to construct a nanocomposite material to be used as a UCL/MRI tracer. The octahedral core-shell UCNP@Fe-MIL-101_NH $\mathrm{N}_{2}$ nanostructures were synthesized using a two-step method. We have found that the core-shell nanocomposites can simultaneously present the NIR optical property of the UCNP cores and the $\mathrm{T}_{2}$-MRI property of the MOF shells. The surface amino groups of the core-shell NPs are conveniently modified with poly(ethylene glycol)-2-amino ethyl ether acetic acid $\left(\mathrm{NH}_{2}\right.$-PEG-COOH) and folic acid (FA), resulting in PEGylated core-shell UCNP@Fe-MIL-101_NH2@PEG-FA nanostructures (UMP-FA for short) that display folic acid moieties. UMP-FAs nanocomposites are tested for $\mathrm{UCL} / \mathrm{T}_{2}$-MRI dual-mode imaging targeted at $\mathrm{KB}$ cells (overexpressing the folate receptor) and KB-tumor bearing mice.

Synthesis of the core-shell UCNP@Fe-MIL-101_NH $\mathrm{NH}_{2}$ nanostructures was performed according to the strategy as shown in Scheme 1. First, monodispersed $\mathrm{Yb}$ and Er doped $\mathrm{NaYF}_{4}$ UCNPs (Y:Yb:Er = 78\%:20\%:2\%, denoted as $\mathrm{NaYF}_{4}: \mathrm{Yb}, \mathrm{Er}$ ) stabilized by oleic acid were synthesized following a literature protocol with slight modifications (Figure 1a). ${ }^{[12]}$ Statistics from transmission electron microscopy (TEM) show that the average diameter of the obtained UCNPs is $40 \mathrm{~nm}$ (Figure 1a, S1e). Analysis by high-resolution TEM (HRTEM) imaging, X-ray powder diffraction (XRD) pattern and energy-dispersive X-ray (EDX) spectrum confirms the composition of the synthesized $\mathrm{NaYF}_{4}: \mathrm{Yb}, \mathrm{Er}$ UCNPs with the hexagonal structure (Figure S1). In the next step the oleic acid was exchanged with polyvinylpyrrolidone (PVP) (Figure 1b, S4a), followed by mixing the ethanol solution of UCNPs, $\mathrm{FeCl}_{3} \cdot 6 \mathrm{H}_{2} \mathrm{O}$ and 2-amino terephthalic acid $\left(\mathrm{NH}_{2}-\mathrm{H}_{2} \mathrm{BDC}\right)$ while gently stirring. Then, 


\section{WILEY-VCH}

the solution was transferred into a $40^{\circ} \mathrm{C}$ oil bath. $1 \mathrm{~h}$ later, core-shell nanostructures with the shell thickness of $\sim 9 \mathrm{~nm}$ were formed (Figure 1c, S2). To reduce agglomeration and increase uniformity, synthesis of the final nanostructures was performed using these pre-synthesized core-shell nanostructures with thin MOF shells (shell thickness of $\sim 9 \mathrm{~nm}$ ) as the seeds. Apparently, the nanostructures exhibit a typical octahedral nanostructure with an average size of $120 \mathrm{~nm}$, where the UCNP cores are eccentric and located at one corner of the nanooctahedrons (Figure 1d, S3). The composition of the core-shell nanostructures is evident from the high-angle annular dark-field scanning transmission electron microscopy (HAADF-STEM) and energy dispersive X-ray (EDX) elemental mapping data (Figure 1e). Evidently, the elements $\mathrm{Y}$ and $\mathrm{F}$ are mainly distributed in the core and that the elements $\mathrm{C}, \mathrm{N}, \mathrm{O}$ and $\mathrm{Fe}$ are homogenously distributed throughout the whole nanostructures, suggesting that the $\mathrm{NaYF}_{4}: \mathrm{Yb}$,Er core is surrounded with an octahedral Fe-MIL-101_NH 2 shell.

The X-ray diffraction (XRD) pattern reveals two sets of characteristic peaks and further confirms that the products are composed of crystalline Fe-MIL-101_NH $\mathrm{N}_{2}$ proved by excellent agreement with the theoretical powder pattern of Cr-MIL-101 at low angles of $1.5^{\circ}-15^{\circ}[10]$ and the hexagonal phase UCNPs at high angles of $15^{\circ}-70^{\circ}$ (Figure 2). Furthermore, the structure and composition of the core-shell products are investigated by Fourier transform infrared spectroscopy (FTIR) as shown in Figure S4b. Compared with the FTIR spectrum of $\mathrm{NH}_{2}$ $\mathrm{H}_{2} \mathrm{BDC}$, the asymmetric vibration band of carboxyl at $1690 \mathrm{~cm}^{-1}$ is shifted to a lower value at $1573 \mathrm{~cm}^{-1}$ appeared in the FTIR spectra of UCNP@Fe-MIL-101_NH $\mathrm{NH}_{2}$ and Fe-MIL-101_NH $\mathrm{N}_{2}$, indicating formation of the coordination bonds between the carboxyl and metal ions in the NMOF shells. ${ }^{[13]}$ The two peaks at around $3480 \mathrm{~cm}^{-1}$ and $3380 \mathrm{~cm}^{-1}$ are attributed to the asymmetric and symmetric stretching absorptions of the primary amine groups. ${ }^{[14]}$ These results verify that the amino-functionalized Fe-MIL-101 shell is successfully coated on the UCNP core. 


\section{WILEY-VCH}

Details of the formation of octahedral core-shell UCNP@Fe-MIL-101_ $\mathrm{NH}_{2}$ nanostructures are elucidated with experiments by varying the reaction time. After the first event of forming a thin shell of about $9 \mathrm{~nm}$ on the surface of UCNPs, the isotropic growth of the shell stops and an octahedral nanostructure begins to appear at one end of the UCNP cores (Figure S5a,b). Then, the octahedral nanostructure tends to self-grow preferentially (Figure S5c) till formation of an eccentric octahedral core-shell nanostructure with the NMOF shell covering the UCNP core (Figure S5d). ${ }^{[15]}$ The formation of such eccentric hybrid nanostructures is attributed to decrease of the interfacial strain between two components of large lattice mismatch. ${ }^{[16]}$

Furthermore, to improve biocompatibility and stability, the core-shell UCNP@Fe-MIL101_ $\mathrm{NH}_{2}$ nanostructures were modified by PEG $\left(\mathrm{NH}_{2}-\mathrm{PEG}-\mathrm{COOH}\right)$ (Figure S6). And both the UCL emission spectrum and the relaxation response of the PEGylated core-shell UCNP@Fe-MIL-101_NH $@$ PEG (UMPs) nanostructures were characterized. From Figure 3a, it can be observed that these nanostructures are well dispersed in water (insert, Figure 3a) and when excited with a $980 \mathrm{~nm}$ laser diode, they exhibit green and red emission from $\mathrm{Er}^{3+}$ ions at the 520, 540, and $654 \mathrm{~nm}$, assigned to the ${ }^{2} \mathrm{H}_{11 / 2} \rightarrow{ }^{4} \mathrm{I}_{15 / 2},{ }^{4} \mathrm{~S}_{3 / 2} \rightarrow{ }^{4} \mathrm{I}_{15 / 2}$, and ${ }^{4} \mathrm{~F}_{9 / 2} \rightarrow{ }^{4} \mathrm{I}_{15 / 2}$ transitions, respectively. ${ }^{[17]}$ The $\mathrm{T}_{2}$-relaxation time of water protons is shortened significantly from about $2047 \mathrm{~ms}$ to $5.6 \mathrm{~ms}$ and the $\mathrm{T}_{2}$-weighted images became darker and darker (insert, Figure 3b) with increasing concentration of Fe $\left(\mathrm{R}_{2}=67.32 \mathrm{mM}^{-1} \mathrm{~s}^{-1}\right.$ at $\left.3 \mathrm{~T}\right)$, which is sufficient for in vivo use. ${ }^{[5]}$ All results suggest that UMPs are a good candidate for UCL/MR imaging. In order to form targeted nanocomposites, the UCNP@Fe-MIL-101_NH $\mathrm{NH}_{2}$ nanostructures were further functionalized with folic acid-modified PEG (UMP-FAs) (Figure S6), which are widely known to be up-regulated in many types of cancer cells. ${ }^{[9]}$ Folate receptors (FRs) are overexpressed in KB tumor cells (human epidermoid carcinoma cell). In order to evaluate the recognition capability and cellular uptake of UMP-FAs, KB cells (FR-positive) and MCF-7 cells (human breast cancer cell, FR-negative) were incubated in PBS buffer containing 100 


\section{WILEY-VCH}

$\mu \mathrm{g} / \mathrm{mL}$ UMP-FAs at $37^{\circ} \mathrm{C}$ For comparison, stained $\mathrm{KB}$ cells were also incubated in the presence of non-conjugated UMPs under identical conditions. As displayed in Figure S7a, KB cells exhibit intense green emissions at 500-560 nm associated with UCL after $2 \mathrm{~h}$ incubation, indicating that UMP-FAs can be rapidly bonded to the surface of the KB cells owing to the high specific interaction between FA on the UMP-FAs and FR on the KB cells. Moreover, with increase of the incubation time to $24 \mathrm{~h}$, strong green fluorescence can be observed both inside the cells and surrounding the cell nucleus (Figure 4a), which implies that UMP-FAs can be uptaken by KB cells into the cytoplasm through the internalization of the bound receptorNP complex as the co-incubation time prolonging. In contrast, there are no obvious uptake in both MCF-7 cells incubated with UMP-FAs (Figure 4b) and KB cells incubated with UMPs (Figure 4c) for $24 \mathrm{~h}$. Furthermore, in vitro $\mathrm{T}_{2}$-weighted MRI of $\mathrm{KB}$ cells incubated with different concentrations of UMP-FAs $(0,125$, and $250 \mu \mathrm{g} / \mathrm{mL})$ were also performed. After coincubation for $24 \mathrm{~h}$, the cells that took up UMP-FAs were precipitated. As shown, the MRI signal at the bottom of tubes in Figure $4 \mathrm{~d}$ became darker with increasing concentration of UMP-FAs, which was attributed to dose-dependent cellular uptake. ${ }^{[8]}$ These results disclose that UMP-FAs can target tumor cells possessing large numbers of folate receptors well. In vitro biocompatibility of UCNP@Fe-MIL-101_NH $\mathrm{NH}_{2}$ with the human oral squamous carcinoma cells was also evaluated utilizing the MTT assay cellular viabilities, which were estimated by incubating KB cells with $300 \mu \mathrm{g} / \mathrm{mL}$ UCNP@Fe-MIL-101_NH $\mathrm{NH}_{2}$ nanostructures for $48 \mathrm{~h}$. The cellular viability was greater than $90 \%$, indicating the negligible cytotoxicity of as-synthesized core-shell nanostructures.

The in vivo targeting ability of the UMP-FAs to FR was evaluated by UCL imaging in mice with KB tumors. Athymic nude mice bearing KB tumor ( 3 weeks post inoculation of $5 \times 10^{6}$ cells on the back, tumor size $8 \sim 10 \mathrm{~mm}$ ) were administered with UMP-FAs and UMPs (1 $\mathrm{mg} / \mathrm{mL}, 200 \mu \mathrm{L}$ ) through tail vein injection. The Balb/c nude mice were imaged using a CALIPER Lumina II in vivo imaging system. A high-power multimode pump laser provided 


\section{WILEY-VCH}

the $980 \mathrm{~nm}$ excitation and UCL signals were collected at 600-700 nm. Impressively, as shown in Figure 5a (left) and Figure S8a, an intense UCL signal is observed in the tumor after intravenous injection of UMP-FAs after $24 \mathrm{~h}$, whereas no significant luminescence signal is discerned in the tumor for the UMPs injected mouse (Figure 5b (left)), confirming the specific in vivo targeting of FRs using UMP-FAs. To further investigate the nanostructure distribution in various organs, ex vivo UCL imaging was also studied. Mice were injected i.v. with UMPFAs and UMPs, respectively, and were sacrificed at the $24 \mathrm{~h}$ time point. The UCL signal (Figure 5a (right) and Figure S8a) is clearly visible in the KB tumor of the mouse injected with UMP-FAs, whereas virtually much weaker signal is seen in the tumor of the mouse injected with UMPs (Figure 5b (right) and Figure S8b), which can be interpreted by limited tumor uptake of the mother particle through the EPR effect. The UCL signal intensity in the ex vivo tumor imaging is clearly attributed to the UCNP cores retained inside the tumor. Both injected nanoprobes are barely seen in kidney and heart, even though some depositions in lung, liver and spleen are observed (Figure 5a, b and Figure S8).

We also investigated the potential of UMP-FAs as a targeted MRI contrast agent in vivo. The $\mathrm{T}_{2}$-weighted MRI was conducted on a KB tumor-bearing mouse using a 3.0T human MRI scanner $24 \mathrm{~h}$ after i.v. injection (1 mg/mL, $200 \mu \mathrm{L}$ for both UMPs and UMP-FAs), and the resulting images are shown in Figure 5c-d. After $24 \mathrm{~h}$, the tumors with the targeted nanostructures show obvious darkening effect. The MRI signal intensity of the KB tumor exhibits a decrease by about 35\%, indicating that UMP-FAs are successfully delivered to KB tumors (Figure S9). Finally, the element Y concentration (from UCNPs) within the tissues was analyzed by inductively coupled plasma atomic emission spectroscopy (ICP-AES) to visualize the localization of UMP-FAs and UMPs. Figure 5e confirms the UMP-FAs uptake by the KB tumors, which uptake is almost 10 times higher than that of UMPs, further revealing that accumulation of the UMP-FAs is mediated by receptor binding. 


\section{WILEY-VCH}

In addition, to investigate the distribution and fate of UMPs nanostructures in vivo with the increasing time, $\mathrm{Y}$ concentrations in the organs (including heart, liver, spleen, lung, and kidney) of mice were analyzed using ICP-MS (Figure 5f). Y element is mainly found in the liver and spleen after $24 \mathrm{~h}$ intravenous injection, whereas $\mathrm{Y}$ concentration in all the organs is declined to lower levels from 1 day to 30 days, which is quite promising for the potential biomedical imaging application. Histology examination was also used to investigate the toxicity and biocompatibility of the materials. As shown in Figure S10, no noticeable tissue damages or any other toxic effect is distinguished on organs, suggesting that there is no obvious damage resulting from the retention profiles of tissues and organs associated with administration of these nanoprobes and they can be safely used for in vivo applications.

In conclusion, we have demonstrated a new efficient method to synthesize novel core-shell UCNP@Fe-MIL-101_NH $\mathrm{NH}_{2}$ nanostructures, in which a $\mathrm{NaYF}_{4}: \mathrm{Yb}, \mathrm{Er}$ NP core is eccentrically coated with amino-functionalized octahedral iron carboxylate NMOF shell. The synthesis results in nanoprobes that possess good water dispersibility, low toxicity, and favorable tissue retention profiles and are easy to conjugate with PEG and FA. Surface-amino functions of the Fe-MIL-101_NH 2 shell are further functionalized with PEG and PEG-FA, resulting in tumortargeted dual-mode UCL/MR imaging agents with high sensitivity, low toxicity and good biocompatibility both in vitro and in vivo. Considering the wide variety, unique properties, and broad applications of both UCNPs and MOFs, we believe the construction strategy of core-shell nanostructures will open up new avenues to integrate advantages of both UCNPs and MOFs for many biological applications, including multimodality imaging, photoregulated drug release, and image-guided targeted cancer therapies.

\section{Supporting Information}

Supporting Information is available from the Wiley Online Library or from the author.

\section{Acknowledgements}

We gratefully thank Prof. Chunhua Yan, Prof. Lingdong Sun, Dr. Yefu Wang and Dr. Hao Dong at Peking University as well as Prof. Yi Hou and Dr. Chunyan Liu at Institute of 


\section{WILEY-VCH}

Chemistry, Chinese Academy of Sciences for their valuable help with in vivo NIR imaging. This work was supported financially by National Key Basic Research Program of China (2014CB931801, Z.Y.T.), National Natural Science Foundation for Distinguished Youth Scholars of China (11425520, C.Y.C.), National Natural Science Foundation of China (21475029 and 91427302, Z.Y.T. and 21371038, Y.L.L.), Instrument Developing Project of the Chinese Academy of Sciences (YZ201311, Z.Y.T.), CAS-CSIRO Cooperative Research Program (GJHZ1503, Z.Y.T.), and "Strategic Priority Research Program" of Chinese Academy of Sciences (XDA09040100, Z.Y.T.).

Received: ((will be filled in by the editorial staff))

Revised: ((will be filled in by the editorial staff)) Published online: ((will be filled in by the editorial staff))

[1] a) M. Vallet-Regi, F. Balas, D. Arcos, Angew. Chem. Int. Ed. 2007, 46, 7548; b) S. Giri, B. G. Trewyn, M. P. Stellmaker, V. S. Y. Lin, Angew. Chem. Int. Ed. 2005, 44, 5038.

[2] P. Horcajada, R. Gref, T. Baati, P. K. Allan, G. Maurin, P. Couvreur, G. Férey, R. E. Morris, C. Serre, Chem. Rev. 2012, 112, 1232.

[3] a) J. Della Rocca, D. Liu, W. B. Lin, Acc. Chem. Res. 2011, 44, 957; b) A. Carné, C. Carbonell, I. Imaz, D. Maspoch, Chem. Soc. Rev. 2011, 40, 291.

[4] a) W. Lin, W. J. Rieter, K. M. L. Taylor, Angew. Chem. Int. Ed. 2009, 48, 650; b) A. M. Spokoyny, D. Kim, A. Sumrein, C. A. Mirkin, Chem. Soc. Rev. 2009, 38, 1218.

[5] a) C. Serre, C. Mellot-Draznieks, S. Surblé, N. Audebrand, Y. Filinch-uk, G. Férey, Science 2007, 315, 1828; b) P. Horcajada, T. Chalati, C. Serre, B. Gillet, C. Sebrie, T. Baati, J. F. Eubank, D. Heurtaux, P. Clayette, C. Kreuz, J. S. Chang, Y. K. Hwang, V. Marsaud, P. N. Bories, L. Cynober, S. Gil, G. Férey, P. Couvreur, R. Gref, Nat. Mater. 2010, 9, 172.

[6] a) G. Chen, H. Qiu, P. N. Prasad, X. Y. Chen, Chem. Rev. 2014, 114, 5161; b) W. Zheng, P. Huang, D. Tu, E. Ma, H. Zhu, X. Chen, Chem. Soc. Rev. 2015, 44, 1379 ; c) R. Naccache, Q. Yu, J. A. Capobianco, Adv. Opt. Mater., 2015, 3, 482 ; d) J. Goldschmidt, S. Fischer, Adv. Opt. Mater., 2015, 3, 510 ; e) R. Martín-Rodríguez, F. T. Rabouw, M. Trevisani, M. Bettinelli, A. Meijerink, Adv. Opt. Mater., 2015, 3, 558 ; f) 


\section{WILEY-VCH}

W. Shao, G. Chen, T. Y. Ohulchanskyy, A. Kuzmin, J. Damasco, H. Qiu, C. Yang, H. Ågren, P. N. Prasad, Adv. Opt. Mater. 2015, 3, 575.

[7] a) J. Gao, G. Liang, J. S. Cheung, Y. Pan,; Y. Kuang, F. Zhao, B. Zhang, X. Zhang, E. Wu, B. Xu, J. Am. Chem. Soc. 2008, 130, 11828; b) C. Zhou, M. Long, Y. Qin,; X. Sun, J. Zheng, Angew. Chem. Int. Ed. 2011, 50, 3168.

[8] a) F. Wang, R. Deng, J. Wang, Q. Wang, Y. Han, H. Zhu, X. Chen, X. Liu, Nat. Mater. 2011, 10, 968; b) W. Fan, B. Shen, W. Bu, F. Chen, K. Zhao, S. Zhang, L. Zhou, W. Peng, Q. Xiao, H. Xing, J. Liu, D. Ni, Q. He, J. Shi, J. Am. Chem. Soc. 2013, 135, 6494.

[9] a) M. T. Kathryn, W. Rieter, W. Lin, J. Am. Chem. Soc. 2008, 130, 14358; b) L. Cheng, K. Yang, Y. Li, J. Chen, C. Wang, M. Shao, S. Lee, Z. Liu, Angew. Chem. Int. Ed. 2011, 50, 7385.

[10] G. Lu, S. Li, Z. Guo, O. K. Farha, B. G. Hauser, X. Qi, Y. Wang, X. Wang, S. Han, X. Liu, J. S. DuChene, H. Zhang, Q. Zhang, X. Chen, J. Ma, S. Loo, W. D. Wei, Y. Yang, J. T. Hupp, F. Huo, Nat. Chem. 2012, 4, 310.

[11] G. Férey, C. Mellot-Draznieks, C. Serre, F. Millange, J. Dutour, S. Surblé, I. Margiolaki, Science, 2005, 309, 2040. (b) K. M. L. Taylor-Pashow, J. D. Rocca, Z. Xie, S. Tran, W. Lin, J. Am. Chem. Soc. 2009, 131, 14261.

[12] a) F. Wang, Y. Han, C. Lim, Y. Lu, J. Wang, J. Xu, H. Chen, C. Zhang, M. Hong, X. Liu, Nature 2010, 463, 1061; b) C. Liu, H. Wang, X. Li, D. Chen, J. Mater. Chem. 2009, 19, 3546.

[13] Y. Li, D. Zhang, Y.-N. Guo, B. Guan, D. Tang, Y. Liu, Q. Huo, Chem. Commun. 2011, 47, 7809.

[14] a) N. J. Johnson, N. M. Sangeetha, J.-C. Boyera, F. C. J. Veggel, Nanoscale 2010, 2, 771; b) M. Zhao, K. Deng, L. He, Y. Liu, G. Li, H. Zhao, Z. Tang, J. Am. Chem. Soc. 2014, 136, 1738. 


\section{WILEY-VCH}

[15] X. Li, R. Cao, D. Sun, W. Bi, Y. Wang, X. Li, M. Hong, Cryst. Growth Des. 2004, 4, 775.

[16] S. E. Habas, H. Lee, V. Radmilovic, G. A. Somorjai, P. Yang, Nat. Mater. 2007, 6, 692.

[17] F. Wang, X. Liu, Chem. Soc. Rev. 2009, 38, 976. 


\section{WILEY-VCH}

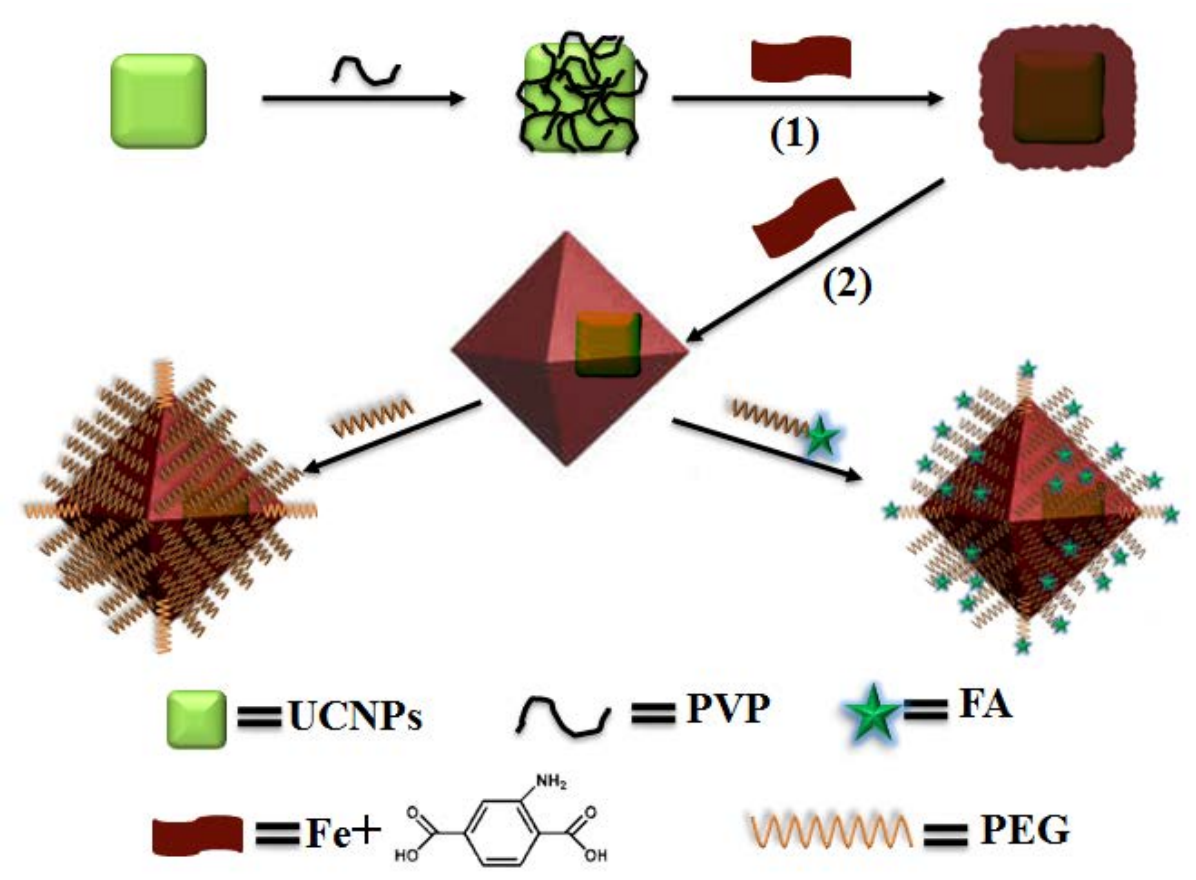

Scheme 1. Strategy for synthesis and functionalization of core-shell UCNP@Fe-MIL101_NH $\mathrm{NH}_{2}$ nanostructures. 


\section{WILEY-VCH}

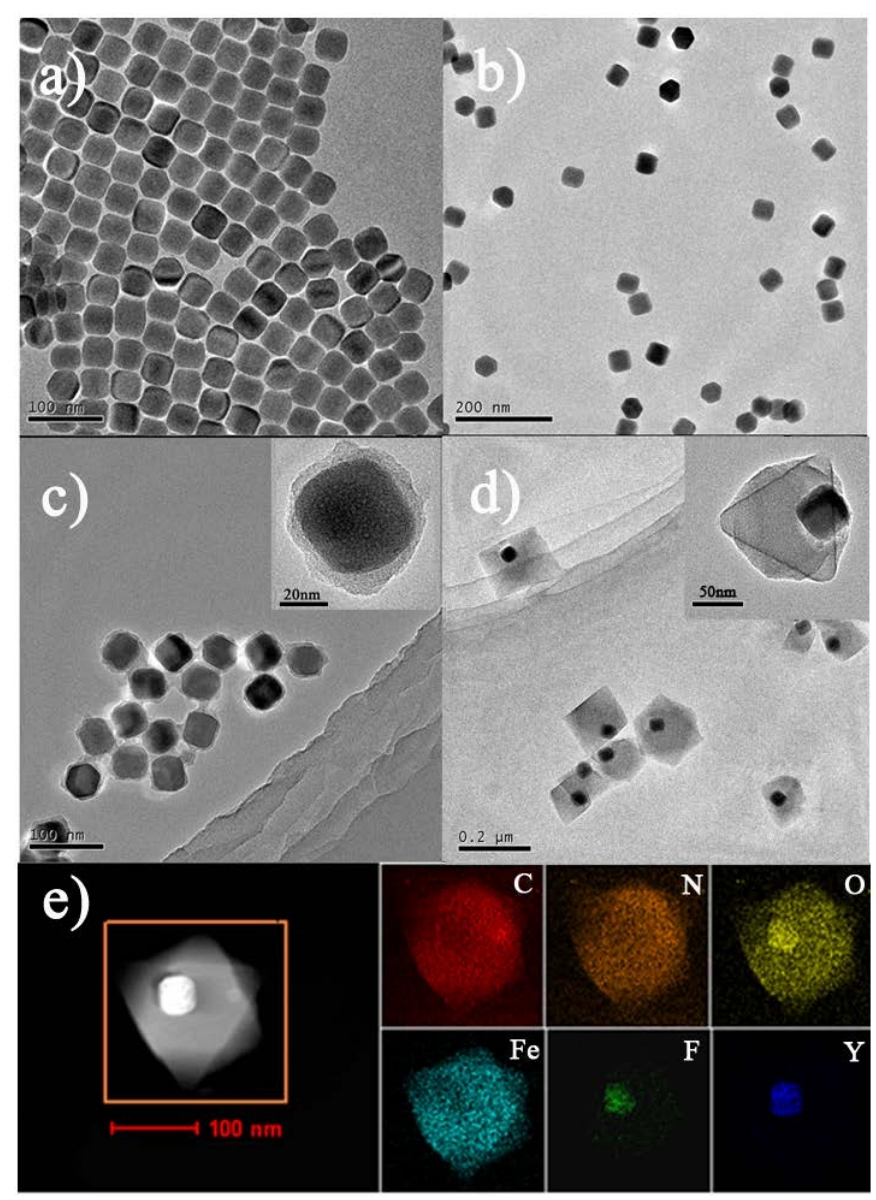

Figure 1. TEM images of (a) oleate-stabilized $\mathrm{NaYF}_{4}$ :Yb,Er UCNPs, (b) PVP-stabilized $\mathrm{NaYF}_{4}: \mathrm{Yb}, \mathrm{Er}$ UCNPs, (c) UCNP@Fe-MIL-101_NH 2 intermediate products with thin MOF shells, and (d) final products with eccentric cores and octahedral MOF shells. (e) HAADFSTEM image and EDX elemental mapping of one core-shell UCNP@Fe-MIL-101_ $\mathrm{NH}_{2}$ nanostructure. 

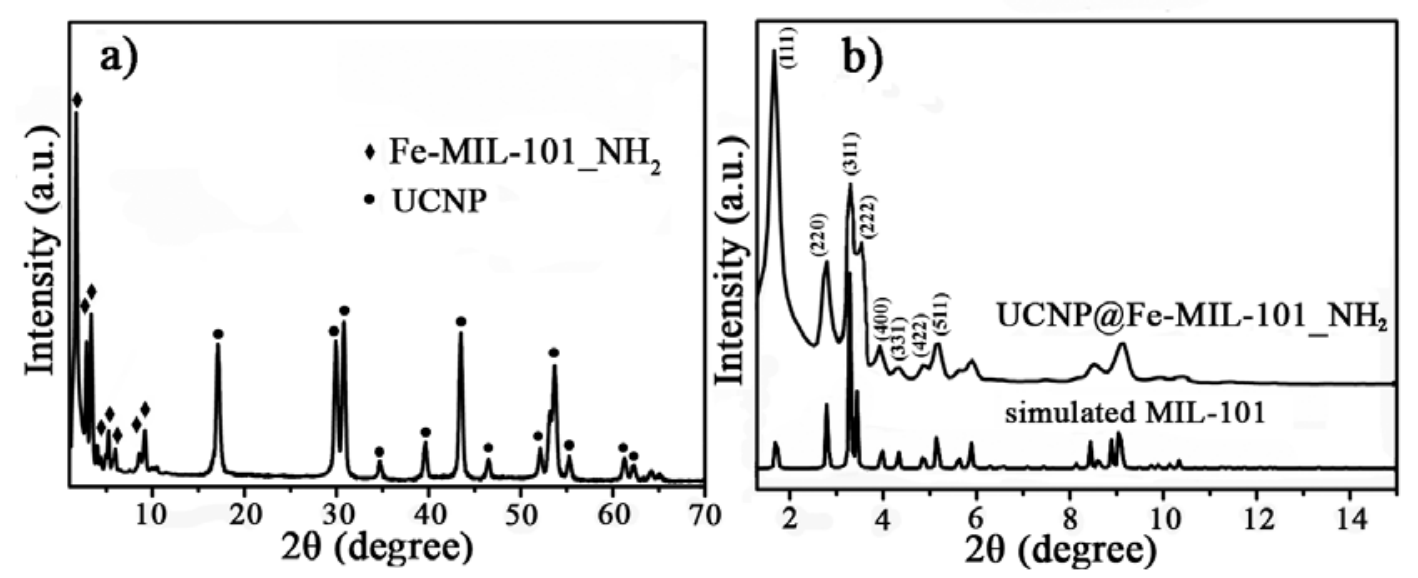

Figure 2. (a) XRD pattern of core-shell UCNP@Fe-MIL-101_NH $\mathrm{NH}_{2}$ nanostructures $\left(1.5^{\circ}-70^{\circ}\right)$.

(b) Magnified XRD pattern at low angles of $1.5^{\circ}$ and $15^{\circ}$ shown in (a) and simulated theoretical pattern of Cr-MIL-101 ${ }^{[10]}$. 


\section{WILEY-VCH}
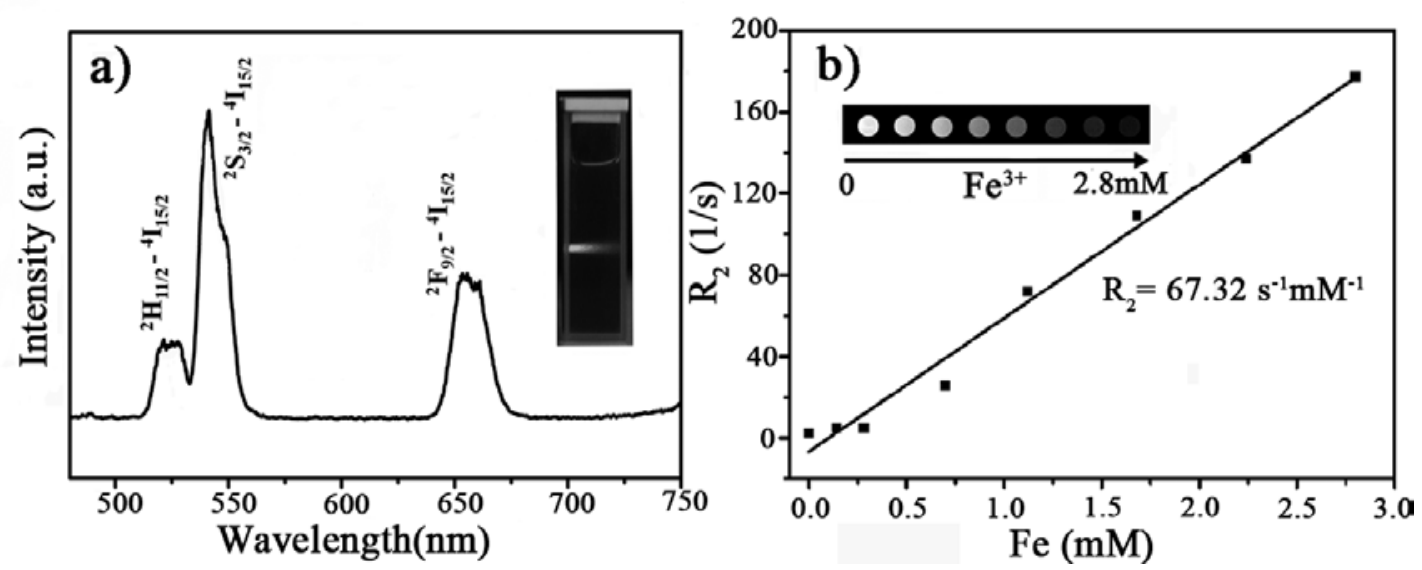

Figure 3. (a) Upconversion emission spectra of UMP nanostructures (Insert is the photograph of corresponding UMP nanostructures dispersed in water under $980 \mathrm{~nm}$ diode excitation). (b) Relaxation rate $R_{2}\left(1 / T_{2}\right)$ versus various molar concentrations of UMP nanostructures at room temperature using a 3T MRI scanner (Insert: $\mathrm{T}_{2}$-weighted MR images of UMPs with varied concentrations). Deionized water (0 mg/mL) was used as the reference. 


\section{WILEY-VCH}
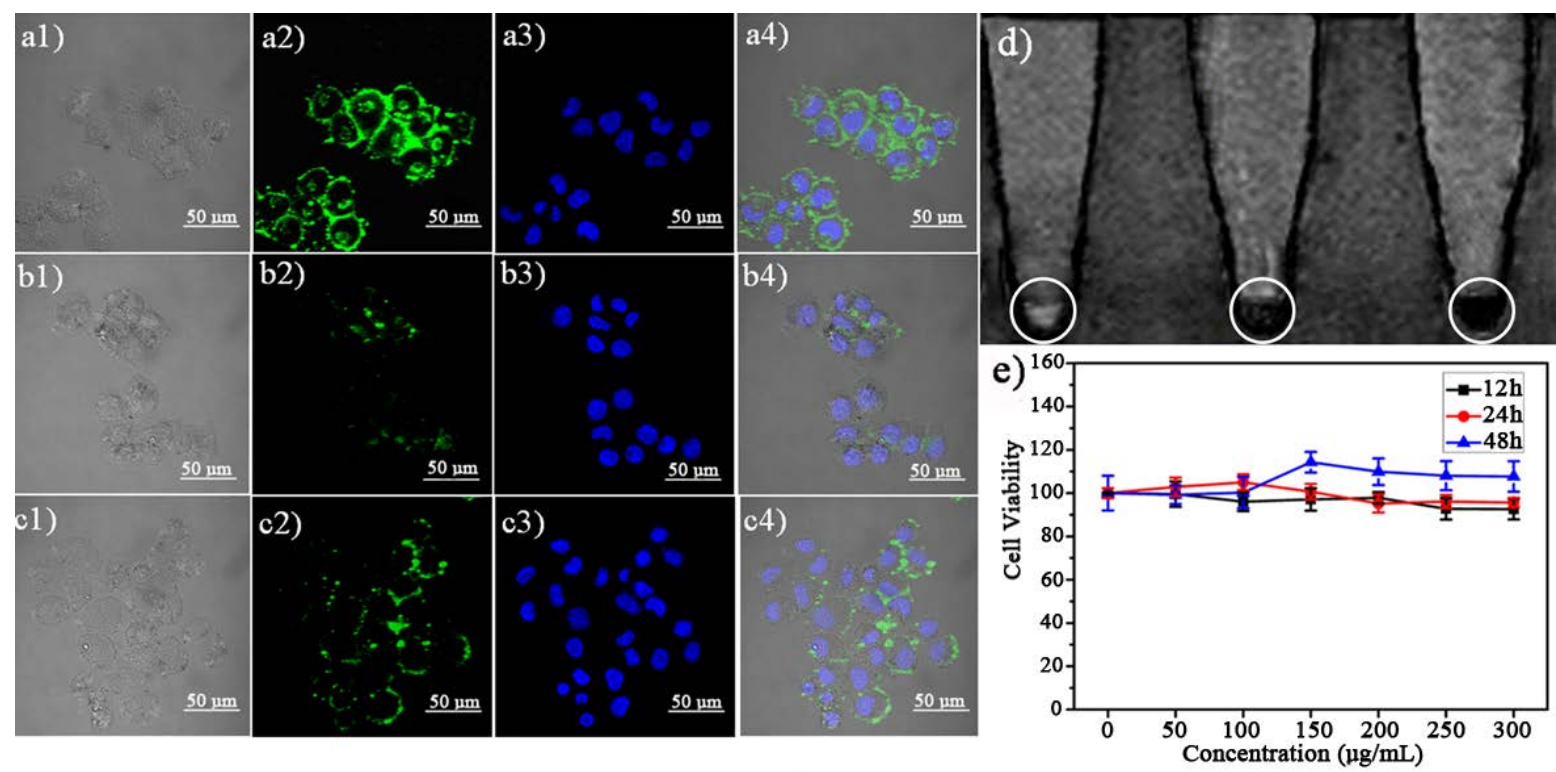

Figure 4. Confocal laser scanning microscopy (CLSM) images of cells irradiated by a CW 980 nm laser: (a) KB cells incubated with UMP-FAs; (b) MCF-7 cells incubated with UMPFAs; and (c) KB cells incubated with UMPs for $24 \mathrm{~h}$. Green channel images were collected at 500-560 nm. The cell nucleus were stained with hochest 33342 (blue fluorescence) and the merge of green channel and brightfield images was also supplied. (d) MRI images of KB cells after co-incubation with UMP-FAs with different concentrations (from left to right: 0, 125 and $250 \mu \mathrm{g} / \mathrm{mL}$ ) for $24 \mathrm{~h}$. Obviously, with increase of the concentration of UMP-FAs added, the $\mathrm{T}_{2}$-MRI signal at the bottom of tubes (marked by the white circles) becomes darker, demonstrating that the MRI signal of KB cells is dose-dependent on UMP-FAs. (e) Cell viability values (\%) estimated by MTT proliferation tests versus incubation concentrations of UCNP@Fe-MIL-101_NH 


\section{WILEY-VCH}

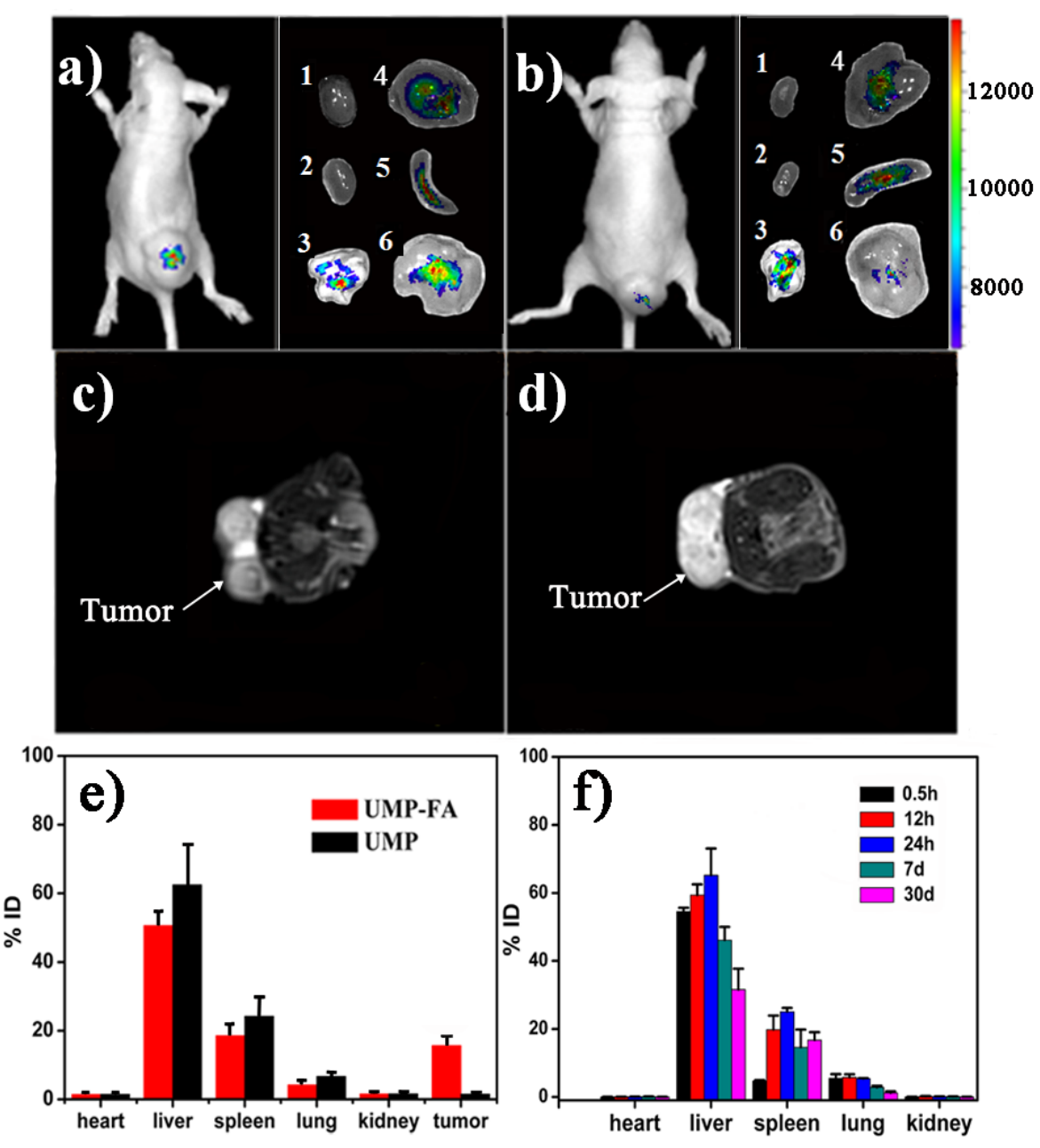

Figure 5. Dual-modal UCL/MR in vivo imaging. Representative UCL imaging of subcutaneous KB tumor-bearing mice (tumor diameter: 8-10 mm) and dissected organs of the mice sacrificed $24 \mathrm{~h}$ after intravenous injection of (a) UMP-FAs (Target) and (b) UMPs (Control). 1, heart; 2, kidney; 3, lung; 4, liver; 5, spleen; 6, KB tumor. All images were acquired under the instrumental conditions (power density $=100 \mathrm{~mW} / \mathrm{cm}^{2}$ on the skin); Representative $\mathrm{T}_{2}$-MRI image of $\mathrm{KB}$ tumor-bearing mice $24 \mathrm{~h}$ after intravenous injection of (c) targeted and (d) non-targeted UMPs. (e) Biodistribution of nanostructures in organs of the mice bearing KB tumor sacrificed $24 \mathrm{~h}$ after intravenous injection of UMP-FAs and UMPs. (f) Biodistribution of UMPs nanostructures in different organs and tissues of mice with intravenous injection for 0.5 h, 12 h, 24 h, 7 days, and 30 days. 